\title{
Association Between Non-Alcoholic Fatty Liver Disease and Bone Turnover Biomarkers in Post-Menopausal Women With Type 2 Diabetes
}

Running title: NAFLD and bone turnover markers in diabetes

Alessandro Mantovani, $\mathrm{MD}^{1}$, Elena Sani, $\mathrm{MD}^{1}$, Angelo Fassio, $\mathrm{MD}^{2}$, Antonio Colecchia, $\mathrm{MD}^{3}$, Ombretta Viapiana, $\mathrm{MD}^{2}$, Davide Gatti, $\mathrm{MD}^{2}$, Luca Idolazzi, $\mathrm{MD}^{2}$, Maurizio Rossini, $\mathrm{MD}^{2}$, Gianluca Salvagno, $\mathrm{MD}^{4}$, Giuseppe Lippi, $\mathrm{MD}^{4}$, Giacomo Zoppini, $\mathrm{MD}^{1}$, Christopher D. Byrne, MB BCh, PhD ${ }^{5,6}$, Enzo Bonora, $\mathrm{MD}^{1}$, Giovanni Targher, $\mathrm{MD}^{1}$

${ }^{1}$ Section of Endocrinology, Diabetes and Metabolism, Department of Medicine, University and Azienda Ospedaliera Universitaria Integrata of Verona, Verona, Italy

${ }^{2}$ Section of Rheumatology, Department of Medicine, University and Azienda Ospedaliera Universitaria Integrata of Verona, Verona, Italy

${ }^{3}$ Gastroenterology Unit, Azienda Ospedaliera Universitaria Integrata of Verona, Verona, Italy

${ }^{4}$ Section of Clinical Biochemistry, University and Azienda Ospedaliera Universitaria Integrata of Verona, Verona, Italy

${ }^{5}$ Nutrition and Metabolism, Faculty of Medicine, University of Southampton, UK

${ }^{6}$ Southampton National Institute for Health Research Biomedical Research Centre, University Hospital Southampton, Southampton General Hospital, Southampton SO16 6YD, UK

Word count: abstract 248; text 3,970 (excluding title page, abstract, references, figure legends and tables); n. 3 Tables; n. 2 Figures

Address of correspondence:

Prof. Giovanni Targher, MD

Section of Endocrinology, Diabetes and Metabolism

University and Azienda Ospedaliera Universitaria Integrata

Piazzale A. Stefani, 1

37126 Verona, Italy

Phone: +39/045-8123748

Fax: +39/045-8027314

E-mail: giovanni.targher@univr.it 


\begin{abstract}
Aim: Information is lacking on the association between non-alcoholic fatty liver disease (NAFLD) and bone mineral density (BMD) or circulating bone turnover biomarkers in post-menopausal women with type 2 diabetes (T2DM).
\end{abstract}

Methods: We recruited 77 white post-menopausal women with T2DM, who consecutively attended our diabetes outpatient service during a 3-month period. Ultrasonography and transient elastography (Fibroscan ${ }^{\circledR}$ ) were used for diagnosing and staging NAFLD. A dual energy X-ray absorptiometry, and serum levels of 25-hydroxyvitamin $\mathrm{D}_{3}[25(\mathrm{OH}) \mathrm{D}]$, parathyroid hormone and multiple bone turnover biomarkers (periostin, sclerostin, dickkopf-related protein-1 [DKK-1], Cterminal telopeptide of type 1 collagen [sCTX], procollagen type $1 \mathrm{~N}$-terminal propeptide [P1NP], receptor activator of nuclear factor-kB ligand [RANKL]) were also measured.

Results: Overall, 10 patients had NAFLD with clinically significant fibrosis (i.e., liver stiffness measurement $>7 \mathrm{kPa}$ ), 52 had NAFLD without fibrosis and 15 patients were free from steatosis. Although the three patient groups had comparable values of BMD, after adjustment for age, waist circumference, HOMA-insulin resistance and serum 25(OH)D levels, patients with NAFLD and significant fibrosis had significantly higher sclerostin levels ( $54.1 \pm 16.4$ vs. $36.1 \pm 11.9$ vs. $42.3 \pm 14.7$ pmol/L) and lower levels of DKK-1 (26.6 \pm 17.8 vs. $49.0 \pm 22.4$ vs. $42.9 \pm 19.4$ pmol/L), RANKL $(0.04 \pm 0.03$ vs. $0.08 \pm 0.06$ vs. $0.11 \pm 0.06 \mathrm{pmol} / \mathrm{L})$ and SCTX (0.16 \pm 0.09 vs. $0.29 \pm 0.17$ vs. $0.40 \pm 0.28 \mathrm{ng} / \mathrm{mL})$ compared to other groups. Serum periostin and P1NP levels did not significantly differ between the groups.

Conclusion: In post-menopausal women with T2DM, the presence of NAFLD and significant fibrosis was associated with a low bone turnover, which may reflect the presence of qualitative bone abnormalities.

Keywords: nonalcoholic fatty liver disease; NAFLD; hepatic fibrosis; bone turnover markers; osteoporosis. 


\section{LIST OF ABBREVIATIONS}

$A L T$, alanine aminotransferase

AST, aspartate aminotransferase

$B M D$, bone mineral density

BMI, body mass index

CKD, chronic kidney disease

CTX, C-terminal telopeptide of type 1 collagen

DEXA, dual energy $X$-ray absorptiometry

DKK-1, dickkopf-related protein-1

eGFR, estimated glomerular filtration rate

GGT, gamma-glutamyltransferase

HOMA-IR, Homeostasis model assessment-insulin resistance

$\mathrm{Hs}-\mathrm{CRP}$, high-sensitivity $\mathrm{C}$ reactive protein

LSM, liver stiffness measurement

NAFLD, nonalcoholic fatty liver disease

$\mathrm{NASH}$, nonalcoholic steatohepatitis

$\mathrm{P} 1 \mathrm{NP}$, procollagen type $1 \mathrm{~N}$-terminal propeptide

PTH, parathyroid hormone

RANKL, receptor activator of nuclear factor-kB ligand

T2DM, type 2 diabetes mellitus

25(OH)D, 25-hydroxyvitamin $\mathrm{D}_{3}$ 


\section{INTRODUCTION}

Non-alcoholic fatty liver disease (NAFLD) has become the predominant cause of chronic liver disease in high-income countries, affecting up to $25-30 \%$ of the general adult population in the Europe and United States, and up to $~ 60-70 \%$ of patients with type 2 diabetes mellitus (T2DM) [13]. Notably, it has been estimated that the global prevalence of NAFLD will rise dramatically over the next decade and will become the most common indication for liver transplantation by 2030 [1].

Over the past 15 years, convincing evidence has emerged that NAFLD is a multisystem disease, which is not only associated with an increased risk of severe liver-related complications, but is also associated with an increased risk of developing extra-hepatic diseases (e.g., cardiovascular disease, chronic kidney disease and colorectal tumours) both in patients with and without T2DM $[4,5]$.

Since there is a close relationship between NAFLD and multiple metabolic, endocrine and inflammatory pathways $[3,4]$, the existence of a potential association between NAFLD and reduced bone mineral density (BMD) has also garnered considerable scientific interest [6]. Osteoporosis is a skeletal disease characterized by low BMD and micro-architectural disruption with a resulting increase in bone fragility and susceptibility to fractures [7]. Osteoporosis is a public health problem due to high cumulative rates of fractures with advancing age. For example, in white populations, about $50 \%$ of women and $20 \%$ of men older than 50 years will have a fragility fracture in their lifetime [7].

A number of observational studies have reported a significant association between imaging-defined NAFLD and reduced BMD so far [6,8-12], especially in Asian men without diabetes. However, there is currently an intense debate in the literature about the existence of this significant association, and a recent small meta-analysis of five cross-sectional studies (involving a total of 1,276 Asian adults) failed to show any significant difference in BMD measurements at the level of femur or lumbar spine between patients with and without NAFLD [13]. In addition, it is currently not known if an association also exists between NAFLD, BMD and circulating levels of bone turnover biomarkers in (white) post-menopausal women or in patients with T2DM, who are two groups at particularly high risk of developing NAFLD and osteoporotic fractures $[1-3,7]$. 
Therefore, we consider that this is a key issue in clinical practice, because it will potentially inform about whether there is an adverse effect of NAFLD on bone health. Furthermore, information about the effect of NAFLD on bone health in the two aforementioned groups of individuals will also help clinicians, who are considering pioglitazone for the treatment of patients with T2DM and nonalcoholic steatohepatitis (NASH), as recommended by recent international guidelines [14].

Thus, the main aim of this cross-sectional study was to examine the existence of a potential association between presence and severity of NAFLD (as detected by ultrasonography and vibration-controlled transient elastography), decreased BMD measurements and abnormal levels of a number of bone turnover biomarkers in white post-menopausal women with T2DM.

\section{MATERIALS AND METHODS}

\section{Patients}

In this pilot study, we enrolled post-menopausal women with established T2DM, who consecutively attended our diabetes outpatient service from October to December 2017. The local Ethics Committee approved the study protocol. All participants gave their written informed consent for participation in this research.

All these female patients met the following inclusion criteria: (a) age $\geq 50$ years; (b) Caucasian; (c) post-menopausal (physiological or surgical); (d) glucose-lowering treatment with diet alone or diet in combination with oral agents (but not with insulin therapy); and (e) no self-reported history of osteoporosis, osteoporotic fractures or treatment with bisphosphonates and other antiosteoporotic agents.

As detailed in Figure 1, we excluded patients with: (a) history of significant alcohol consumption (i.e., >20 grams of alcohol per day) or known causes of chronic liver disease (e.g., virus, drugs, autoimmunity, hemochromatosis); (b) history of cirrhosis of any etiology, cancer and end-stage renal disease (defined as estimated glomerular filtration rate $<15 \mathrm{ml} / \mathrm{min} / 1.73 \mathrm{~m}^{2}$ or chronic dialysis); (c) history of overt hyperthyroidism or hypothyroidism; and (d) treatment with hormone replacement therapy or steroids. 
Overall, 77 white post-menopausal women with T2DM (mean \pm SD age: $72 \pm 8$ years; body mass index (BMI): $29 \pm 5 \mathrm{~kg} / \mathrm{m}^{2}$; hemoglobin A1c (HbA1c): $\left.52 \pm 8 \mathrm{mmol} / \mathrm{mol}\right)$ were included in the final analysis.

\section{Clinical and Laboratory Data}

$\mathrm{BMI}$ was measured as kilograms divided by the square of height in meters. Waist circumference was measured at the midpoint between the lowest rib and the iliac crest. Blood pressure was measured with a standard sphygmomanometer after the patient had been seated quietly for at least 5 minutes. Patients were considered to have hypertension if their blood pressure was $\geq 140 / 90 \mathrm{mmHg}$ or if they were taking any anti-hypertensive drug. Information on the type of menopause (physiological or surgical), smoking and use of medications was obtained from all patients via interviews during medical examinations.

Venous blood samples were drawn in the morning after an overnight fast. Measurements of serum glucose, lipids, creatinine (measured using a Jaffè rate blanked and compensated assay), electrolytes (sodium, potassium, calcium, phosphorus), aspartate aminotransferase (AST), alanine aminotransferase (ALT), gamma-glutamyltransferase (GGT), high-sensitivity C reactive protein (hsCRP) and other biochemical blood parameters were obtained using standard laboratory procedures at the central Laboratory of our hospital. HbA1c was measured using the high-performance liquid chromatography analyzer Tosoh-G7 (Tosoh Bioscience Inc., Tokyo, Japan). Fasting insulin was measured using a chemiluminescent immunoassay method (LIAISON, DiaSorin, Saluggia, Italy). Homeostasis model assessment (HOMA-IR) score was used for estimating insulin resistance. Glomerular filtration rate (e-GFR $\mathrm{MDRD}$ ) was estimated using the four-variable Modification of Diet in Renal Disease (MDRD) study equation [15]. Urinary albumin excretion was assessed with an immuno-nephelometric assay (Beckman-Coulter IMMAGE; Beckman-Coulter Instruments, Fullerton, CA, USA) on a morning spot urine sample and expressed as the albumin-to-creatinine ratio (ACR); abnormal albuminuria was defined as a urinary $A C R \geq 30 \mathrm{mg} / \mathrm{g}$ creatinine.

Chronic kidney disease (CKD) was defined as the presence of eGFR $\mathrm{MDRD}<60 \mathrm{~mL} / \mathrm{min} / 1.73 \mathrm{~m}^{2}$ with or without accompanying abnormal albuminuria. Pre-existing history of ischemic heart disease (IHD) or stroke was defined as a documented history of myocardial infarction, angina or coronary revascularizations or ischemic stroke. Pre-existing history of peripheral artery disease was based on medical history and examination (e.g., intermittent claudication, rest pain or lower-extremity 
revascularizations), and was then confirmed by reviewing hospital medical records of patients, including radiologic imaging results. Presence of diabetic retinopathy (diagnosed with fundoscopy after pupillary dilation) was also recorded.

\section{Serum bone turnover biomarkers}

Serum samples were collected from all patients at the time of study recruitment, centrifuged, separated and stored at $-80^{\circ} \mathrm{C}$ until measurement. An expert laboratory technician, who was blinded to patients' clinical details, measured 25-hydroxyvitamin $\mathrm{D}_{3}[25(\mathrm{OH}) \mathrm{D}]$, intact parathyroid hormone (PTH) and a number of indirect bone turnover biomarkers, such as sclerostin, periostin, Cterminal telopeptide of type 1 collagen (sCTX), procollagen type $1 \mathrm{~N}$-terminal propeptide (P1NP), dickkopf-related protein 1 (DKK-1) and soluble ligand of the kappa-B factor activator receptor (RANKL). Specifically, 25(OH)D, PTH, sCTX and P1NP were measured using the IDS-ISYS MultiDiscipline Automated Analyzer (Immunodiagnostic System, Boldon, UK) employing immunochemiluminescent technology, whilst DKK-1, sclerostin, periostin and RANKL were measured by commercially available enzyme immunoassay ELISA kits (Biomedica Medizinprodukte, Vienna, Austria) on the fully automated microplate analyzer Personal LAB (Adaltis, Rome, Italy). The intraassay coefficients of variation (CV), in our laboratory, were 4\% for P1NP (inter-assay CV 6\%), 3\% for SCTX (inter-assay CV 7\%), 5\% (inter-assay CV 6.9\%) for sclerostin, 7\% for Dkk-1 (inter-assay CV 8.2\%), 2.7\% for PTH (inter-assay CV 5.5\%), 6\% for 25(OH)D (inter-assay CV 9\%), and $<10 \%$ (inter-assay CV $<12 \%)$ for both RANKL and periostin, respectively.

\section{Dual energy X-ray absorptiometry}

A DEXA scan was performed in all patients using the GE Lunar iDXA system (GE Healthcare Lunar, Madison, WI, USA) by a single expert operator, who was blinded to patients' clinical details, including results of liver ultrasonography and transient elastography.

The axial densitometry was performed to obtain BMD measurements $\left(\mathrm{g} / \mathrm{cm}^{2}\right)$ at the level of both lumbar spine (L1-L4) and femur (neck and total). For each skeletal site, both $T$-scores and Z-scores were calculated. Osteoporosis was defined as a $T$-score <-2.5 SD [7]. The total body densitometry was also performed for measuring body composition, i.e. bone mineral content (BMC), lean and fat masses. 


\section{Liver ultrasonography and liver stiffness measurement}

Liver ultrasonography and liver stiffness measurement (LSM) were performed by a single expert physician, who was blinded to all patients' biochemical and clinical data, including also DEXA measurements.

Hepatic steatosis was diagnosed by ultrasonography (using an Esaote MyLab 70 ultrasound with a 4 $\mathrm{MHz}$ probe) according to specific ultrasonographic characteristics, such as diffuse hyperechogenicity of the liver relative to kidneys, ultrasonographic beam attenuation, and poor visualization of intra-hepatic vessel borders and diaphragm [16]. It is known that ultrasonography has a good sensitivity and specificity for detecting moderate-to-severe hepatic steatosis, whereas its sensitivity is lower when the hepatic fat infiltration on liver histology is $<20 \%-30 \%[16,17]$. The ultrasonographic degree of hepatic steatosis was expressed with a semi-quantitative scale (i.e., absent, mild, moderate or severe).

LSMs were performed using vibration-controlled transient elastography (Fibroscan ${ }^{\circledR}$ Echosens, Paris, France) with a M probe. An expert physician performed LSMs in all patients after at least six hours of fasting and after liver ultrasonography. Further details of the technical background and examination procedures have been previously described [18]. Briefly, each measurement was considered adequate when including at least ten valid measurements for each patient, with a success rate $>60 \%$ and variability of measurements (IQR) $<30 \%$ of the median [18]. The presence of clinically significant hepatic fibrosis was defined by the presence of a LSM $>7 \mathrm{kPa}[17,19]$.

\section{Statistical analysis}

Data are expressed as means $\pm S D$, medians (and interquartile ranges) or percentages. Based on the presence/absence of hepatic steatosis on ultrasonography and severity of hepatic fibrosis on Fibroscan ${ }^{\circledR}$, all patients were classified in the following three groups: (a) patients without hepatic steatosis (no-NAFLD group); (b) patients with NAFLD (hepatic steatosis) and clinically significant fibrosis; and (c) patients with NAFLD with no significant fibrosis.

Differences for clinical, biochemical and DEXA characteristics were firstly assessed using either the one-way analysis of variance (ANOVA) for continuous variables (skewed variables were logarithmically transformed before analysis) or the chi-squared test for categorical variables. A post- 
hoc Tukey's HSD (Honestly Significant Difference) procedure was used for pairwise comparisons of means between groups. Analysis of covariance (ANCOVA) was used to test the inter-group differences in circulating levels of bone turnover biomarkers and DEXA measurements after adjustment for potential confounding factors. We performed four ANCOVA models that were adjusted for age and waist circumference (adjusted model 1), or for age, waist circumference and HOMA-IR score (model 2), or for age, waist circumference, HOMA-IR score and serum 25(OH)D levels (model 3) or, finally, for age, waist circumference, HbA1c and 25(OH)D levels (model 4). The covariates included in these models were selected as potential confounding factors based on their significance in univariable analyses.

A $p$-value $<0.05$ was considered statistically significant. Statistical analyses were performed using STATA software, version 14.2 (STATA, College Station, Tx, USA).

\section{RESULTS}

Among the 77 white post-menopausal women with T2DM included in the study, 62 (80.5\%) patients met the diagnostic criteria for NAFLD, whereas 15 (19.5\%) patients did not have hepatic steatosis. Among those with NAFLD, 36 patients had mild hepatic steatosis and 26 had moderate-to-severe steatosis on ultrasonography. Ten patients with NAFLD had hepatic steatosis with clinically significant fibrosis (i.e. LSM >7 kPa), whereas 52 had hepatic steatosis without significant fibrosis.

Table 1 shows the main clinical and biochemical characteristics of patients stratified by the presence/absence of hepatic steatosis on ultrasonography and severity of fibrosis on Fibroscan ${ }^{\circledR}$. Patients with NAFLD, irrespective of the presence of significant fibrosis, were more likely to be younger, more centrally obese, more insulin resistant, and had a lower duration of menopause than their counterparts without NAFLD. Patients with NAFLD and significant fibrosis also had higher levels of $\mathrm{HbA} 1 \mathrm{c}$ and serum liver enzymes (although remaining largely within the reference ranges of the local laboratory) compared to other two patient groups.

Conversely, no significant differences were observed in BMI, blood pressure, full blood count, albumin, lipid profile, smoking, comorbid conditions (such as IHD, ischemic stroke, peripheral artery disease or diabetic retinopathy) as well as in the current use of various medications, such as oral 
glucose-lowering agents, lipid-lowering agents, antiplatelet drugs, proton-pump inhibitors or antihypertensive agents (including diuretics). Regarding oral glucose-lowering drugs, the use of metformin, sulphonylureas, acarbose, pioglitazone, incretin mimetics or sodium/glucose cotransporter-2 (SGLT2) inhibitors was comparable among the three patient groups.

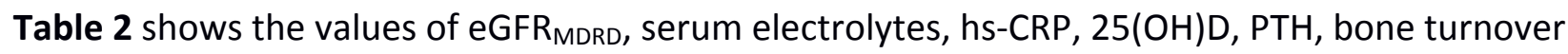
biomarkers as well as BMD and other DEXA measurements in patients stratified by the presence/absence of NAFLD on ultrasonography and severity of hepatic fibrosis on Fibroscan ${ }^{\circledR}$.

Although patients with NAFLD and significant fibrosis were more frequently treated with oral vitamin $D_{3}$ supplementation, they had significantly lower serum $25(\mathrm{OH}) \mathrm{D}$ values than the other two groups. The significant differences in serum 25(OH)D values persisted after adjustment for age and waist circumference, but were no longer statistically significant after further adjustment for HOMAIR score.

With regards to circulating levels of bone turnover biomarkers, patients with NAFLD and significant fibrosis had higher serum sclerostin and lower values of SCTX, RANKL and DKK-1 compared to both NAFLD patients without fibrosis and those without steatosis (as assessed by post-hoc Tukey's HSD tests). Notably, the differences in these bone turnover biomarkers remained significant even after adjusting for age, waist circumference, HOMA-IR score, HbA1c or 25(OH)D levels. Conversely, no differences were found in eGFR $\mathrm{MDRD}_{\mathrm{N}}$, hs-CRP, electrolytes, periostin, P1NP and PTH levels among the three groups of patients.

With regards to DEXA measurements (as also shown in Table 2), no significant differences were found in BMC, lumbar and femoral BMD, T-scores and Z-scores, as well as total fat and lean masses among the three patient groups.

All the aforementioned findings remained unchanged even when the results were additionally adjusted for years of menopause, or when the only patient treated with pioglitazone or those treated with SGLT-2 inhibitors ( $n=5)$ were excluded from statistical analyses (data not shown). 
Figure 2 shows the prevalence of osteoporosis (i.e., $T$-score <-2.5 SD) in patients stratified by the presence of NAFLD on ultrasonography and severity of hepatic fibrosis on Fibroscan ${ }^{\circledR}$. No significant differences could be observed in the prevalence of osteoporosis at the level of the lumbar spine or femur among the groups.

Table 3 shows the values of eGFR ${ }_{\text {MDRD }}$, serum electrolytes, hs-CRP, 25(OH)D, PTH and bone turnover biomarkers in patients classified according to the ultrasonographic severity of hepatic steatosis (absent vs. mild vs. moderate-severe steatosis). Notably, no significant differences were found in any of the measured bone turnover markers as well as in BMC and lumbar/femoral BMD measurements among the groups (data not shown).

\section{DISCUSSION}

The novel findings of this exploratory study are as follows: (1) post-menopausal type 2 diabetic women with NAFLD and significant fibrosis had significantly higher serum sclerostin and lower DKK1, RANKL and SCTX levels (along with decreased serum 25(OH)D concentrations) compared to both NAFLD patients without significant fibrosis and those without steatosis; (2) the significant differences in these bone turnover biomarkers were only slightly attenuated after adjustment for age, serum 25(OH)D, waist circumference, HOMA-IR score or HbA1c; (3) no differences were found in BMC, lumbar/femoral BMD measurements or prevalence of osteoporosis among the three groups of patients; and (4) no differences were also found in any of the measured bone turnover biomarkers when patients were stratified by severity of hepatic steatosis on ultrasonography.

To our knowledge, this is the first cross-sectional study specifically aimed at examining the association between presence and severity of NAFLD, BMD measurements and a large panel of circulating bone turnover biomarkers in a sample of consecutive post-menopausal women with T2DM.

At present, controversy still exists in the literature regarding the existence of any association between NAFLD and decreased BMD or osteoporosis in adults. Although some cross-sectional studies reported a significant association between NAFLD and reduced femoral BMD [6,8-11,19], 
most of these studies included Asian individuals [8-10], and some of these studies could only find a significant association in men, but not in women $[8,10,20]$. Again, a recent small meta-analysis of five cross-sectional studies (totaling nearly 1,300 Asian individuals) failed to show any significant difference in BMD measurements between patients with and without NAFLD [13]. Paradoxically, a recent cross-sectional study of South Korean healthy individuals reported a negative association between NAFLD and femoral neck BMD in men, but a positive association was instead observed between NAFLD and lumbar spine BMD in post-menopausal women, thus suggesting a possible sexrelated differential effect of NAFLD on BMD [21]. In line with this finding, two cross-sectional retrospective studies of Chinese middle-aged individuals showed that the presence of NAFLD was independently associated with a recent history of osteoporotic fractures in men, but not in women $[12,22]$.

In this study, we found that patients with NAFLD and significant fibrosis had decreased serum 25(OH)D levels (a known risk factor for osteoporosis), although these patients took higher daily dosages of oral vitamin $D_{3}$ compared to the other two patient groups. A plausible explanation for this finding is that patients with more advanced liver disease could have a reduced enzymatic efficiency of the 25 -hydroxylation of vitamin $D_{3}$. However, some experimental data showed that specific enzymatic reactions, including vitamin $D_{3}$ hydroxylation, were maintained in patients with biopsy-proven NASH [23]. In addition, emerging evidence suggests that lower serum 25(OH)D values per se may predict the progression of NAFLD (rather than being a simple epiphenomenon of the liver disease), as lower serum 25(OH)D concentrations may worsen both hepatic insulin resistance and histologic features of NAFLD [24]. That said, it is likely that these aspects may co-exist all together and, thus, explain the lower serum 25(OH)D levels we observed in patients with NAFLD and significant fibrosis. However, more prospective and mechanistic studies are needed to better elucidate this issue.

Notably, we found significant differences in serum DKK-1, RANKL, SCTX and sclerostin levels in patients with NAFLD and significant fibrosis compared to the other two groups of patients that persisted after adjusting for age, waist circumference, HOMA-insulin resistance and serum 25(OH)D levels (and despite comparable values of BMD). In particular, one of the most interesting finding of this study is the presence of markedly lower levels of DKK-1, a protein acting as antagonist of WNT/ $\beta$-catenin pathway, among patients with NAFLD and significant fibrosis. Experimental data 
indicates that hepatocytes are not able to release adequate quantities of DKK-1 when liver fibrosis occurs [25]. The WNT/ $\beta$-catenin pathway may regulate both the osteoblastic and osteoclastic activity, mainly through the modulation of the RANKL to osteoprotegerin ratio (26). In this study, we did not measure serum osteoprotegerin, but we found decreased RANKL levels (likely to be secondary to lower DKK-1 levels) in patients with NAFLD and significant fibrosis, which might also be accountable for the lower levels of SCTX (a marker of bone resorption) observed in our patients. Lower levels of DKK-1 should be also expected to be associated with an increase in serum P1NP levels (a marker of bone formation). In fact, bone resorption and bone formation are two processes closely inter-related $[27,28]$, and every factor interfering with one of these two processes (i.e., disease or treatment) is inevitably going to a consensual change of the other one as well [28]. However, the increase in serum sclerostin levels, i.e., another inhibitor of the WNT/ $\beta$-catenin pathway acting similarly to DKK-1, we observed in patients with NAFLD and significant fibrosis might partly explain the lack of the expected increase in serum P1NP levels. We believe that the higher serum sclerostin levels of patients with NAFLD and significant fibrosis may reflect the counterregulatory activity of WNT/ $\beta$-catenin signaling, as also previously reported in other investigations [29-31]. However, more research is needed to confirm these findings, and to clarify the underlying mechanisms linking NAFLD (with varying amounts of hepatic fibrosis) to alterations of the WNT/ $\beta$ catenin pathway.

Interestingly, we failed to find significant differences in serum bone turnover biomarkers after stratifying patients by the severity of hepatic steatosis on ultrasound. We believe that this information may be clinically relevant, as indicating that the severity of hepatic fibrosis, rather than hepatic steatosis, may contribute to the observed abnormalities in serum bone turnover biomarkers in our patients. In line with this finding, convincing evidence now indicates that the severity of hepatic fibrosis, rather other histologic features, is the strongest predictor for the development of hepatic and extra-hepatic complications in NAFLD $[1,2,14,17,19]$.

Collectively, the results of our study suggest that the presence of NAFLD and significant hepatic fibrosis is associated with a state of low bone turnover in post-menopausal women with T2DM, which may contribute to enhance bone fragility. It is plausible to assume that serum bone turnover biomarkers have the potential to be stronger predictors of long-term risk of fractures than BMD measurements in these patients. Indeed, in our study most BMD measurements were within the 
normal range, in agreement with the current body of evidence showing that BMD underestimates the fracture risk in patients with T2DM, thus making risk assessment challenging (27). Accumulating evidence now indicates that diabetic bone disease is characterized by a low bone turnover, and that patients with T2DM have a higher long-term risk of bone fractures, although they have similar or slightly higher BMD measurements compared to age- and sex-adjusted non-diabetic controls [27,31-34]. Although most therapies for osteoporosis seem to be safe in T2DM, the presence of a low bone turnover status might decrease the rational behind the use of antiresorptive agents, while it might support the use of anabolic therapies (i.e., teriparatide). However, no randomized controlled trials have been conducted in patients with T2DM at present.

Our study has some important limitations that should be mentioned. First, the cross-sectional design may limit our ability to establish causality and temporality of the observed associations. Second, since we included only Caucasian female outpatients with T2DM, who were treated with diet and/or oral glucose-lowering agents (but not with insulin therapy), our findings may not necessarily be generalizable to other diabetic populations of different ethnicity. Third, the number of patients with NAFLD and significant fibrosis was relatively low, so that we could not make an extensive adjustment for potential confounders in multivariable regression models. Fourth, although the diagnosis of NAFLD and severity of hepatic fibrosis were based on the use of ultrasonography and Fibroscan, i.e., two imaging techniques widely used in clinical practice, we did not perform liver biopsy, which is the "reference standard" for diagnosing and staging NAFLD $[14,17]$. However, we believe that it would have been hazardous to perform liver biopsies in these T2DM patients with normal or only slightly higher serum liver enzymes. Finally, in our study, we could not perform direct measurements of bone microstructure by using bone tissue biopsies or a high-resolution peripheral quantitative computed tomography scan.

Despite these limitations, our study has important strengths, including the consecutive enrollment of the population studied, the measurement of a large number of different bone resorption and formation biomarkers, the completeness of the database, the adjustment for serum 25(OH)D levels and important metabolic risk factors, and the exclusion of patients with documented osteoporosis, those with important comorbid conditions (e.g., advanced kidney disease, cirrhosis or cancer) or those taking medications known to interfere with bone metabolism. We believe that including patients with these conditions might have confounded the interpretation of data. 
In conclusion, this cross-sectional studies shows for the first time that the presence of NAFLD and significant fibrosis in white post-menopausal women with T2DM was independently associated with a low bone turnover, as reflected by decreased serum levels of DKK-1, RANKL and SCTX. Even though DXA measurements are not able to detect a significant decrease in BMD in patients with NAFLD and clinically significant fibrosis, the presence of a low bone turnover might mirror the existence of qualitative bone abnormalities, which increase risk of bone fragility in these patients. Further studies are needed to confirm these findings, and provide new insights into the association between NAFLD, low bone turnover and fracture risk in post-menopausal women with T2DM.

Disclosure Statement: The Authors have no potential conflicts of interest to disclose.

Funding Sources: GT is supported in part by grants from the University School of Medicine of Verona, Verona, Italy. CDB is supported in part by grants from the Southampton National Institute for Health Research Biomedical Research Centre.

Acknowledgements: The authors would like to thank Dr. Eliana Amato for the measurements of serum bone turnover biomarkers and the LURM (Laboratorio Universitario di Ricerca Medica) Research Center, University of Verona, where these biochemical analyses were performed.

\section{REFERENCES}

1. Lonardo A, Bellentani S, Argo CK, Ballestri S, Byrne CD, Caldwell SH, et al. Epidemiological modifiers of non-alcoholic fatty liver disease: Focus on high-risk groups. Dig Liver Dis 2015; 47: 997-1006.

2. Younossi Z, Anstee QM, Marietti M, Hardy T, Henry L, Eslam M, et al. Global burden of NAFLD and NASH: trends, predictions, risk factors and prevention. Nat Rev Gastroenterol Hepatol 2018; 15: 11-20.

3. Targher G, Lonardo A, Byrne CD. Nonalcoholic fatty liver disease and chronic vascular complications of diabetes mellitus. Nat Rev Endocrinol 2018; 14: 99-114.

4. Byrne CD, Targher G. NAFLD: a multisystem disease. J Hepatol 2015; 62: S47-S64. 
5. Adams LA, Anstee QM, Tilg H, Targher G. Non-alcoholic fatty liver disease and its relationship with cardiovascular disease and other extra-hepatic diseases. Gut 2017; 66: 1138-53.

6. Targher G, Lonardo A, Rossini M. Nonalcoholic fatty liver disease and decreased bone mineral density: is there a link? J Endocrinol Invest 2015; 38: 817-25.

7. Black DM, Rosen CJ. Postmenopausal osteoporosis. N Engl J Med 2016; 374; 254-62.

8. Yang HJ, Shim SG, Ma BO, Kwak JY. Association of nonalcoholic fatty liver disease with bone mineral density and serum osteocalcin levels in Korean men. Eur J Gastroenterol Hepatol 2016; 28: 338-44.

9. Kim G, Kim KJ, Rhee Y, Lim SK. Significant liver fibrosis assessed using liver transient elastography is independently associated with low bone mineral density in patients with non-alcoholic fatty liver disease. PLoS One 2017; 12: e0182202.

10. Cui R, Sheng H, Rui XF, Cheng XY, Sheng CJ, Wang JY, et al. Low bone mineral density in chinese adults with nonalcoholic Fatty liver disease. Int J Endocrinol 2013; 2013:396545.

11. Umehara T. Nonalcoholic fatty liver disease with elevated alanine aminotransferase levels is negatively associated with bone mineral density: cross-sectional study in US adults. PLoS One 2018; 13: e0197900.

12. Li M, Xu Y, Xu M, Ma L, Wang T, Liu Y, et al. Association between nonalcoholic fatty liver disease (NAFLD) and osteoporotic fracture in middle-aged and elderly Chinese. J Clin Endocrinol Metab 2012; 97: 2033-38.

13. Upala S, Jaruvongvanich V, Wijarnpreecha K, Sanguankeo A. Nonalcoholic fatty liver disease and osteoporosis: a systematic review and meta-analysis. J Bone Miner Metab 2017; 35: 685-93.

14. Chalasani N, Younossi Z, Lavine JE, Charlton M, Cusi K, Rinella M, et al. The diagnosis and management of nonalcoholic fatty liver disease: practice guidance from the American Association for the Study of Liver Diseases. Hepatology 2018; 67: 328-37.

15. Levey AS, Bosch JP, Lewis JB, Greene T, Rogers N, Roth D. Modification of Diet in Renal Disease Study Group. A more accurate method to estimate glomerular filtration rate from serum creatinine: a new prediction equation. Ann Intern Med 1999; 130: 461-70.

16. Hernaez R, Lazo M, Bonekamp S, Kamel I, Brancati FL, Guallar E, et al. Diagnostic accuracy and reliability of ultrasonography for the detection of fatty liver: a meta-analysis. Hepatology 2011; 54: 1082-90.

17. Byrne CD, Patel J, Scorletti E, Targher G. Tests for diagnosing and monitoring non-alcoholic fatty liver disease in adults. BMJ 2018; 362: k2734. 
18. Colecchia A, Montrone L, Scaioli E, Bacchi-Reggiani ML, Colli A, Casazza G, et al. Measurement of spleen stiffness to evaluate portal hypertension and the presence of esophageal varices in patients with HCV-related cirrhosis. Gastroenterology 2012; 143: 646-54.

19. Tapper EB, Loomba R. Noninvasive imaging biomarker assessment of liver fibrosis by elastography in NAFLD. Nat Rev Gastroenterol Hepatol 2018; 15: 274-82.

20. Xia MF, Lin HD, Yan HM, Bian H, Chang XX, Zhang LS, et al. The association of liver fat content and serum alanine aminotransferase with bone mineral density in middle-aged and elderly Chinese men and postmenopausal women. J Transl Med 2016; 14: 11.

21. Lee SH, Yun JM, Kim SH, Seo YG, Min H, Chung E, et al. Association between bone mineral density and nonalcoholic fatty liver disease in Korean adults. J Endocrinol Invest 2016; 39: 132936.

22. Wang Y, Wen G, Zhou R, Zhong W, Lu S, Hu C, et al. Association of nonalcoholic fatty liver disease with osteoporotic fractures: a cross-sectional retrospective study of Chinese individuals. Front Endocrinol (Lausanne) 2018; 9: 408.

23. Barchetta I, Carotti S, Labbadia G, Gentilucci UV, Muda AO, Angelico F, et al. Liver vitamin D receptor, CYP2R1, and CYP27A1 expression: relationship with liver histology and vitamin D3 levels in patients with nonalcoholic steatohepatitis or hepatitis C virus. Hepatology 2012; 56: 2180-87.

24. Targher G, Scorletti E, Mantovani A, Byrne CD. Nonalcoholic fatty liver disease and reduced serum vitamin $D_{3}$ levels. Metab Syndr Relat Disord 2013; 11: 217-28.

25. Polyzos SA, Anastasilakis AD, Kountouras J, Makras P, Papatheodorou A, Kokkoris P, et al. Circulating sclerostin and Dickkopf-1 levels in patients with nonalcoholic fatty liver disease. J Bone Miner Metab 2016; 34: 447-56.

26. Fassio A, Rossini M, Viapiana O, Idolazzi L, Vantaggiato E, Benini C, et al. New strategies for the prevention and treatment of systemic and local bone loss: from pathophysiology to clinical application. Curr Pharm Dis 2017; 23: 6241-50.

27. Napoli N, Chandran M, Pierroz DD, Abrahamsen B, Schwartz AV, Ferrari SL; IOF Bone and Diabetes Working Group. Mechanisms of diabetes mellitus-induced bone fragility. Nat Rev Endocrinol 2017; 13: 208-19.

28. Adami S, Kanis JA. Assessment of involutional bone loss: methodological and conceptual problems. J Bone Miner Res 1995; 10: 511-17. 
29. Gatti D, Viapiana O, Fracassi E, Idolazzi L, Dartizio C, Povino MR, et al. Sclerostin and DKK1 in post-menopausal osteoporosis treated with denosumab. J Bone Miner Res 2012; 27: 2259-63.

30. Viapiana O, Fracassi E, Troplini S, Idolazzi L, Rossini M, Adami S, et al. Sclerostin and DKK1 in primary hyperparathyroidism. Calcif Tissue Int 2013; 92: 324-29.

31. Miao CG, Yang YY, He $X$, Huang $C$, Huang $Y$, Zhang $L$, et al. Wnt signaling in liver fibrosis: progress, challenges and potential directions. Biochimie 2013; 95: 2326-35.

32. Fan Y, Wei F, Lang Y, Liu Y. Diabetes mellitus and risk of hip fractures: a meta-analysis. Osteoporos Int 2015; 27: 219-28.

33. Furst JR, Bandeira LC, Fan WW, Agarwal S, Nishiyama KK, McMahon DJ, et al. Advanced glycation end-products and bone material strength in type 2 diabetes. J Clin Endocrinol Metab 2016; 101: 2502-10.

34. Hygum K, Starup-Linde J, Harsløf T, Vestergaard P, BL Langdahl. Diabetes mellitus, a state of low bone turnover - a systematic review and meta-analysis. Eur J Endocrinol 2017; 176: R137-157. 
Table 1. Clinical and biochemical characteristics of white postmenopausal women with type 2 diabetes stratified by presence and severity of NAFLD (using both ultrasonography and Fibroscan ${ }^{\circledR}$ ).

\begin{tabular}{|c|c|c|c|c|}
\hline & $\begin{array}{l}\text { Patients with no hepatic } \\
\text { steatosis }(n=15)\end{array}$ & $\begin{array}{l}\text { Patients with hepatic } \\
\text { steatosis alone }(n=52)\end{array}$ & $\begin{array}{l}\text { Patients with steatosis and } \\
\text { significant fibrosis }(n=10)\end{array}$ & $P$ \\
\hline Age (years) & $76.8 \pm 7$ & $70.7 \pm 7$ & $71.8 \pm 9$ & $<0.05$ \\
\hline Weight (Kg) & $66.4 \pm 12$ & $74.1 \pm 13$ & $72.4 \pm 10$ & 0.10 \\
\hline $\mathrm{BMI}\left(\mathrm{Kg} / \mathrm{m}^{2}\right)$ & $27.1 \pm 5$ & $29.6 \pm 5$ & $29.6 \pm 4$ & 0.22 \\
\hline Waist circumference $(\mathrm{cm})$ & $90.2 \pm 11$ & $99.1 \pm 13$ & $101.7 \pm 10$ & $<0.05$ \\
\hline Diabetes duration (years) & $15(7-21)$ & $10(6-16)$ & $11(7-15)$ & 0.19 \\
\hline Type of menopause (physiological) (\%) & 80 & 96 & 90 & 0.12 \\
\hline Menopause duration (years) & $27.1 \pm 7$ & $21.3 \pm 7$ & $21.7 \pm 9$ & $<0.05$ \\
\hline Current smokers (\%) & 20 & 10 & 20 & 0.37 \\
\hline Family history of cardiovascular disease (\%) & 40 & 23 & 40 & 0.30 \\
\hline Family history of diabetes (\%) & 47 & 59 & 60 & 0.65 \\
\hline Systolic blood pressure (mmHg) & $142 \pm 13$ & $136 \pm 14$ & $139 \pm 18$ & 0.32 \\
\hline Diastolic blood pressure (mmHg) & $76 \pm 10$ & $76 \pm 8$ & $75 \pm 11$ & 0.77 \\
\hline White blood cell count $\left(\times 10^{9} / \mathrm{L}\right)$ & $6.9 \pm 1.3$ & $7.1 \pm 1.4$ & $7.3 \pm 1.4$ & 0.36 \\
\hline Hemoglobin (g/L) & $128 \pm 10$ & $132 \pm 10$ & $132 \pm 11$ & 0.35 \\
\hline Platelet count $(x 100,000 / \mathrm{mmc})$ & $245 \pm 43$ & $256 \pm 56$ & $232 \pm 52$ & 0.38 \\
\hline Fasting glucose $(\mathrm{mmol} / \mathrm{L})$ & $6.9 \pm 1.1$ & $7.1 \pm 1.6$ & $8.0 \pm 1.7$ & 0.13 \\
\hline Hemoglobin A1c (mmol/mol) & $50.7 \pm 4.7$ & $51.1 \pm 8.6$ & $58.4 \pm 10.4$ & $<0.05$ \\
\hline Total cholesterol (mg/dL) & $162 \pm 21$ & $162 \pm 35$ & $151 \pm 25$ & 0.56 \\
\hline LDL-cholesterol (mg/dL) & $76 \pm 17$ & $83 \pm 32$ & $69 \pm 1$ & 0.31 \\
\hline HDL-cholesterol (mg/dL) & $66 \pm 14$ & $58 \pm 12$ & $61 \pm 20$ & 0.15 \\
\hline Triglycerides (mg/dL) & $119 \pm 62$ & $125 \pm 54$ & $125 \pm 66$ & 0.61 \\
\hline AST (IU/L) & $21 \pm 5$ & $22 \pm 7$ & $29 \pm 10$ & $<0.05$ \\
\hline ALT (IU/L) & $11 \pm 3$ & $14 \pm 7$ & $18 \pm 9$ & $<0.05$ \\
\hline GGT (IU/L) & $12(10-17)$ & $16(13-28)$ & $32(16-63)$ & $<0.05$ \\
\hline Albumin $(\mathrm{g} / \mathrm{dL})$ & $44.2 \pm 2$ & $44.4 \pm 3$ & $44.3 \pm$ & 0.93 \\
\hline Fasting insulin (mU/L) & $3.3(2.7-6.5)$ & $7.9(4.5-12.4)$ & $11.7(4.5-22.5)$ & $<0.05$ \\
\hline HOMA-IR score & $1.1(0.7-1.9)$ & $2.3(1.3-4.1)$ & $3.8(1.8-8.3)$ & $<0.05$ \\
\hline Abnormal albuminuria (\%) & 7 & 8 & 10 & 0.95 \\
\hline Hypertension (\%) & 93 & 75 & 90 & 0.20 \\
\hline Ischemic heart disease (\%) & 13 & 12 & 10 & 0.96 \\
\hline Ischemic stroke (\%) & 0 & 2 & 10 & 0.26 \\
\hline Peripheral artery disease (\%) & 20 & 8 & 10 & 0.38 \\
\hline Diabetic retinopathy, any degree (\%) & 20 & 4 & 20 & 0.09 \\
\hline Metformin (\%) & 80 & 81 & 90 & 0.77 \\
\hline Sulphonylureas (\%) & 40 & 25 & 20 & 0.44 \\
\hline Pioglitazone (\%) & 0 & 2 & 0 & 0.78 \\
\hline DPP-4 inhibitors (\%) & 40 & 23 & 40 & 0.30 \\
\hline GLP-1 analogues (\%) & 20 & 10 & 10 & 0.53 \\
\hline Acarbose (\%) & 13 & 6 & 10 & 0.61 \\
\hline SGLT-2 inhibitors (\%) & 0 & 8 & 10 & 0.50 \\
\hline Anti-platelet drugs (\%) & 60 & 37 & 60 & 0.15 \\
\hline Beta-blockers (\%) & 27 & 31 & 30 & 0.95 \\
\hline ARB/ACE-inhibitors (\%) & 87 & 56 & 90 & 0.09 \\
\hline Calcium-channel blockers (\%) & 7 & 19 & 20 & 0.49 \\
\hline Diuretics (\%) & 40 & 33 & 40 & 0.82 \\
\hline Nitrates (\%) & 7 & 3.8 & 0 & 0.70 \\
\hline Statins (\%) & 87 & 73 & 80 & 0.52 \\
\hline Fibrates (\%) & 0 & 2 & 10 & 0.26 \\
\hline Omega-3 fatty acids (\%) & 0 & 6 & 10 & 0.51 \\
\hline Proton-pump inhibitors (\%) & 20 & 23 & 30 & 0.84 \\
\hline
\end{tabular}

Sample size, $n=77$. Data are expressed as means \pm SD, medians and IQR (in parenthesis) or percentages. Differences among the groups were tested by the one-way ANOVA for continuous variables (skewed variables were logarithmically transformed before analysis) and the chi-squared test for categorical variables. NB: A multiple-comparison post-hoc Tukey's HSD test showed that there were significant differences in age, waist circumference, menopause duration, serum insulin and HOMA-IR score in patients without steatosis vs. either patients with hepatic steatosis alone or those with steatosis and significant fibrosis; and there were significant differences in serum liver enzymes and HbA1c levels in patients with steatosis and significant fibrosis vs. either patients with hepatic steatosis alone or those without steatosis.

Abbreviations: ACE, angiotensin-converting-enzyme; ALT, alanine aminotransferase; ARB, angiotensin II receptor blocker; AST, aspartate aminotransferase; BMI, body mass index; DPP-4, dipeptidyl peptidase-4; GGT, gamma-glutamyltransferase; GLP-1, glucagon-like peptide-1; HOMA-IR, homeostasis model assessment-insulin resistance; SGLT-2, sodium/glucose cotransporter-2.

Note: Hypertension was defined as blood pressure $\geq 140 / 90 \mathrm{mmHg}$ or drug treatment. Abnormal albuminuria was defined as urinary ACR $\geq 30 \mathrm{mg} / \mathrm{g}$. 
Table 2. Serum levels of hs-C-reactive protein, estimated glomerular filtration rate, electrolytes, 25-hydroxyvitamin $\mathrm{D}_{3}$, parathyroid hormone and bone turnover biomarkers, as well as DEXA parameters in white postmenopausal women with type 2 diabetes stratified by presence and severity of NAFLD (using both ultrasonography and Fibroscan ${ }^{\circledR}$ ).

\begin{tabular}{|c|c|c|c|c|c|c|c|c|}
\hline & $\begin{array}{l}\text { Patients with no } \\
\text { hepatic steatosis } \\
\text { ( } n=15 \text { ) }\end{array}$ & \begin{tabular}{|l} 
Patients with \\
hepatic steatosis \\
alone ( $n=52)$
\end{tabular} & $\begin{array}{l}\text { Patients with steatosis } \\
\text { and significant fibrosis } \\
\text { ( } n=10)\end{array}$ & $\begin{array}{l}\text { Unadjusted } \\
P \text { value for } \\
\text { trend }\end{array}$ & \begin{tabular}{|l|} 
Adjusted \\
$P$ value \\
(model 1) \\
\end{tabular} & \begin{tabular}{|l} 
Adjusted \\
$P$ value* \\
(model 2)
\end{tabular} & \begin{tabular}{|l|} 
Adjusted \\
$P$ value \\
(model 3)
\end{tabular} & \begin{tabular}{|l} 
Adjusted \\
$P$ value \\
(model 4)
\end{tabular} \\
\hline hs-C reactive protein $(\mathrm{mg} / \mathrm{L})$ & $0.7(0.4-3.2)$ & $1.9(1.1-2.4)$ & $3.1(1.1-5.5)$ & 0.06 & 0.66 & 0.71 & 0.74 & 0.79 \\
\hline e-GFR ${ }_{\text {MDRD }}\left(\mathrm{mL} / \mathrm{min} / 1.73 \mathrm{~m}^{2}\right)$ & $78.4 \pm 16$ & $87.6 \pm 19$ & $85.3 \pm 21$ & 0.28 & 0.38 & 0.54 & 0.45 & 0.34 \\
\hline Sodium (mmol/L) & $144 \pm 3.5$ & $143 \pm 2.7$ & $144 \pm 3.8$ & 0.77 & 0.79 & 0.87 & 0.97 & 0.84 \\
\hline Potassium (mmol/L) & $4.4 \pm 0.4$ & $4.3 \pm 0.3$ & $4.3 \pm 0.3$ & 0.93 & 0.94 & 0.77 & 0.76 & 0.87 \\
\hline Calcium (mmol/L) & $2.3 \pm 0.1$ & $2.4 \pm 0.1$ & $2.3 \pm 0.1$ & 0.92 & 0.91 & 0.81 & 0.84 & 0.92 \\
\hline Phosphorus (mmol/L) & $1.1 \pm 0.1$ & $1.1 \pm 0.1$ & $1.1 \pm 0.2$ & 0.53 & 0.31 & 0.27 & 0.26 & 0.14 \\
\hline Vitamin D supplementation (\%) & 31 & 46 & 33 & 0.49 & 0.35 & 0.23 & ND & ND \\
\hline Dosage of vitamin D supplementation (IU/day) & $880(800-3300)$ & $1000(800-1200)$ & $1500(1000-3300)$ & $<0.05$ & $<0.05$ & 0.10 & ND & ND \\
\hline 25-hydroxyvitamin $\mathrm{D}_{3}(\mathrm{ng} / \mathrm{mL})$ & $32.9 \pm 8.8$ & $31.9 \pm 13.4$ & $22.2 \pm 7.7$ & $<0.05$ & $<0.05$ & 0.09 & ND & ND \\
\hline PTH $(\mathrm{pg} / \mathrm{mL})$ & $31.3 \pm 14.6$ & $35.4 \pm 20.4$ & $36.2 \pm 18.8$ & 0.74 & 0.87 & 0.81 & 0.70 & 0.78 \\
\hline SCTX (ng/mL) & $0.40 \pm 0.28$ & $0.29 \pm 0.17$ & $0.16 \pm 0.09$ & $<0.005$ & $<0.01$ & $<0.05$ & $<0.05$ & 0.053 \\
\hline Sclerostin (pmol/L) & $42.3 \pm 14.7$ & $36.1 \pm 11.9$ & $54.1 \pm 16.4$ & $<0.005$ & $<0.005$ & $<0.005$ & $<0.005$ & $<0.01$ \\
\hline Periostin $(\mathrm{ng} / \mathrm{mL})$ & $96.9 \pm 24.1$ & $87.9 \pm 26.7$ & $80.9 \pm 28.4$ & 0.24 & 0.49 & 0.72 & 0.64 & 0.45 \\
\hline DKK-1 (pmol/L) & $42.9 \pm 19.4$ & $48.0 \pm 22.4$ & $26.6 \pm 17.8$ & $<0.005$ & $<0.005$ & $<0.005$ & $<0.005$ & $<0.01$ \\
\hline RANKL (pmol/L) & $0.11 \pm 0.06$ & $0.08 \pm 0.06$ & $0.04 \pm 0.03$ & $<0.01$ & $<0.01$ & $<0.05$ & $<0.05$ & $<0.01$ \\
\hline P1NP (ng/mL) & $58.4 \pm 26.8$ & $51.9 \pm 17.7$ & $42.9 \pm 15.6$ & 0.12 & 0.29 & 0.37 & 0.41 & 0.49 \\
\hline Fat mass $(\mathrm{g})$ & $25798 \pm 8544$ & $31443 \pm 9192$ & $31386 \pm 6094$ & 0.09 & 0.88 & 0.66 & 0.59 & 0.76 \\
\hline Lean mass (g) & $37608 \pm 4970$ & $40694 \pm 4435$ & $39313 \pm 4887$ & 0.08 & 0.46 & 0.26 & 0.20 & 0.33 \\
\hline BMC (g) & $1958 \pm 288$ & $2107 \pm 300$ & $2267 \pm 397$ & 0.10 & 0.71 & 0.38 & 0.59 & 0.46 \\
\hline Lumbar BMD $\left(\mathrm{g} / \mathrm{cm}^{2}\right)$ & $1.05 \pm 0.15$ & $1.06 \pm 0.17$ & $1.15 \pm 0.18$ & 0.26 & 0.33 & 0.29 & 0.57 & 0.59 \\
\hline Lumbar T score & $-1.06 \pm 1.23$ & $-0.96 \pm 1.44$ & $-0.21 \pm 1.55$ & 0.27 & 0.34 & 0.30 & 0.56 & 0.42 \\
\hline Lumbar Z score & $0.71 \pm 1.18$ & $0.38 \pm 1.39$ & $1.20 \pm 1.21$ & 0.19 & 0.24 & 0.18 & 0.57 & 0.47 \\
\hline Femoral neck BMD $\left(\mathrm{g} / \mathrm{cm}^{2}\right)$ & $0.78 \pm 0.13$ & $0.84 \pm 0.13$ & $0.87 \pm 0.14$ & 0.21 & 0.70 & 0.63 & 0.79 & 0.81 \\
\hline Femoral neck T score & $-1.83 \pm 0.91$ & $-1.45 \pm 0.96$ & $-1.18 \pm 0.98$ & 0.23 & 0.69 & 0.60 & 0.77 & 0.80 \\
\hline Femoral neck Z score & $0.20 \pm 0.79$ & $0.10 \pm 0.84$ & $0.43 \pm 0.92$ & 0.53 & 0.60 & 0.47 & 0.63 & 0.68 \\
\hline Femoral hip BMD $\left(\mathrm{g} / \mathrm{cm}^{2}\right)$ & $0.85 \pm 0.14$ & $0.93 \pm 0.14$ & $0.97 \pm 0.18$ & 0.11 & 0.65 & 0.64 & 0.85 & 0.81 \\
\hline Femoral hip T score & $-1.19 \pm 1.10$ & $-0.62 \pm 1.12$ & $-0.27 \pm 1.36$ & 0.12 & 0.64 & 0.63 & 0.84 & 0.82 \\
\hline Femoral hip Z score & $0.65 \pm 0.97$ & $0.68 \pm 0.95$ & $1.13 \pm 1.21$ & 0.40 & 0.60 & 0.47 & 0.62 & 0.68 \\
\hline
\end{tabular}

Sample size, $n=77$. Data are expressed as means \pm SD, medians and IQR (in parenthesis) or percentages. Differences among the groups were tested by the one-way ANOVA for continuous variables (skewed variables were logarithmically transformed before analysis) and the chi-squared test for categorical variables. NB: A multiple-comparison post-hoc Tukey's HSD test showed that there were significant differences in serum 25-hydroxyvitamin D, sCTX, sclerostin, DKK-1 and RANKL levels in patients with hepatic steatosis and significant fibrosis vs. either patients with hepatic steatosis alone or those without hepatic steatosis, whereas no significant differences were found in serum bone turnover biomarkers, 25-hydroxyvitamin D or DEXA measurements between patients with hepatic steatosis alone and those without hepatic steatosis.

Note: "model 1: adjusted for age and waist circumference; " $m$ model 2: adjusted for age, waist circumference and HOMA-IR score; ${ }^{* *}$ model 3: adjusted for age, waist circumference, HOMA-IR score and 25(OH)D levels; and ${ }^{* * *}$ model 4: adjusted for age, waist circumference, $\mathrm{HbA1c}$ and $25(\mathrm{OH}) \mathrm{D}$ levels.

Abbreviations: BMC, bone mineral content; BMD, bone mineral density; DKK-1, dickkopf-related protein 1; ND, not determined; P1NP, procollagen type $1 \mathrm{~N}$-terminal propeptide; PTH, parathyroid hormone; RANKL, receptor activator of nuclear factor-KB ligand; SCTX, serum carboxy-terminal collagen I; e-GFRMDRD, glomerular filtration rate estimated by using the Modification of Diet in Renal Diseases study equation. 
Table 3. Serum levels of hs-C-reactive protein, estimated glomerular filtration rate, electrolytes, 25-hydroxyvitamin $D_{3}$, parathyroid hormone and bone turnover biomarkers in white postmenopausal women with type 2 diabetes stratified by the severity of hepatic steatosis on ultrasonography.

\begin{tabular}{|c|c|c|c|c|c|c|c|c|}
\hline & $\begin{array}{l}\text { Patients with no } \\
\text { hepatic steatosis } \\
(\mathrm{n}=15)\end{array}$ & \begin{tabular}{|l|} 
Patients with \\
mild hepatic \\
steatosis $(n=36)$
\end{tabular} & \begin{tabular}{|l|} 
Patients with \\
moderate-severe \\
steatosis ( $n=26$ ) \\
\end{tabular} & $\begin{array}{l}\text { Unadjusted } \\
P \text { value for } \\
\text { trend }\end{array}$ & \begin{tabular}{|l} 
Adjusted \\
$P$ value \\
(model 1) $^{*}$ \\
\end{tabular} & \begin{tabular}{|l|} 
Adjusted \\
P value* \\
(model 2) \\
\end{tabular} & \begin{tabular}{|l} 
Adjusted \\
$P$ value** \\
(model 3) $^{*}$
\end{tabular} & \begin{tabular}{|l|} 
Adjusted \\
$P$ value \\
(model 4)
\end{tabular} \\
\hline hs-C reactive protein $(\mathrm{mg} / \mathrm{L})$ & $0.7(0.4-3.2)$ & $2.2(0.7-5.0)$ & $1.9(0.6-3.9)$ & 0.14 & 0.40 & 0.57 & 0.56 & 0.42 \\
\hline e-GFR ${ }_{\text {MDRD }}\left(\mathrm{mL} / \mathrm{min} / 1.73 \mathrm{~m}^{2}\right)$ & $78.4 \pm 16$ & $83.1 \pm 17$ & $83.8 \pm 25$ & 0.69 & 0.52 & 0.53 & 0.35 & 0.29 \\
\hline Sodium (mmol/L) & $144 \pm 3.5$ & $144 \pm 2.6$ & $143 \pm 4.3$ & 0.69 & 0.69 & 0.65 & 0.66 & 0.70 \\
\hline Potassium (mmol/L) & $4.4 \pm 0.4$ & $4.3 \pm 0.3$ & $4.3 \pm 0.3$ & 0.94 & 0.89 & 0.85 & 0.85 & 0.89 \\
\hline Calcium $(\mathrm{mmol} / \mathrm{L})$ & $2.3 \pm 0.1$ & $2.3 \pm 0.1$ & $2.3 \pm 0.1$ & 0.91 & 0.92 & 0.97 & 0.97 & 0.94 \\
\hline Phosphorus (mmol/L) & $1.1 \pm 0.1$ & $1.1 \pm 0.2$ & $1.1 \pm 0.1$ & 0.93 & 0.94 & 0.94 & 0.94 & 0.92 \\
\hline Vitamin D supplementation (\%) & 31 & 48 & 39 & 0.65 & 0.76 & 0.78 & ND & ND \\
\hline Dosage of vitamin D supplementation (IU/day) & $0.7(0.4-3.2)$ & $1000(800-1100)$ & $900(800-2025)$ & 0.49 & 0.42 & 0.46 & ND & ND \\
\hline 25-hydroxyvitamin $\mathrm{D}_{3}(\mathrm{ng} / \mathrm{mL})$ & $32.9 \pm 8.8$ & $29.5 \pm 14.1$ & $28.3 \pm 10.8$ & 0.43 & 0.41 & 0.60 & ND & ND \\
\hline PTH $(\mathrm{pg} / \mathrm{mL})$ & $31.3 \pm 14.6$ & $38.2 \pm 20.2$ & $32.4 \pm 9.9$ & 0.45 & 0.44 & 0.31 & 0.23 & 0.35 \\
\hline sCTX (ng/mL) & $0.40 \pm 0.28$ & $0.26 \pm 0.17$ & $0.29 \pm 0.24$ & 0.52 & 0.46 & 0.43 & 0.46 & 0.54 \\
\hline Sclerostin $(\mathrm{pmol} / \mathrm{L})$ & $42.3 \pm 14.7$ & $44.3 \pm 16.9$ & $42.6 \pm 22.2$ & 0.23 & 0.10 & 0.10 & 0.13 & 0.16 \\
\hline \begin{tabular}{|l} 
Periostin $(\mathrm{ng} / \mathrm{mL})$ \\
\end{tabular} & $96.9 \pm 24.1$ & $85.9 \pm 25.7$ & $96.3 \pm 32.6$ & 0.26 & 0.24 & 0.30 & 0.28 & 0.23 \\
\hline DKK-1 (pmol/L) & $42.9 \pm 19.4$ & $45.5 \pm 22.7$ & $46.4 \pm 21.1$ & 0.83 & 0.87 & 0.83 & 0.89 & 0.94 \\
\hline RANKL (pmol/L) & $0.11 \pm 0.06$ & $0.07 \pm 0.06$ & $0.07 \pm 0.05$ & 0.22 & 0.23 & 0.22 & 0.27 & 0.29 \\
\hline P1NP (ng/mL) & $58.4 \pm 26.8$ & $50.6 \pm 17.6$ & $50.8 \pm 23.8$ & 0.24 & 0.21 & 0.19 & 0.22 & 0.27 \\
\hline
\end{tabular}

Sample size, $n=77$. Data are expressed as means \pm SD, medians and IQR (in parenthesis) or percentages. Differences among the groups were tested by the one-way ANOVA for continuous variables (skewed variables were logarithmically transformed before analysis) and the chi-squared test for categorical variables.

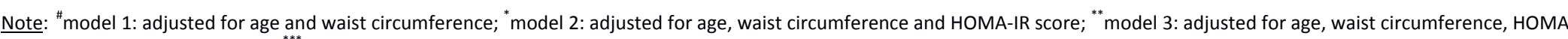
IR score and 25(OH)D levels; and ${ }^{* * *}$ model 4: adjusted for age, waist circumference, $\mathrm{HbA1c}$ and $25(\mathrm{OH}) \mathrm{D}$ levels.

Abbreviations: DKK-1, dickkopf-related protein 1; ND, not determined; P1NP, procollagen type $1 \mathrm{~N}$-terminal propeptide; PTH, parathyroid hormone; RANKL, receptor activator of nuclear

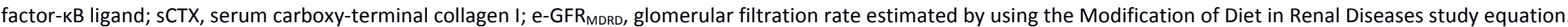




\section{FIGURE LEGENDS}

Figure 1. Flow-chart of the study design.

Figure 2. Prevalence of osteoporosis at the level of the lumbar spine and femur (defined as a $T$ score $<-2.5 \mathrm{SD}$ ) in white post-menopausal type 2 diabetic women stratified by presence and severity of NAFLD (as detected by ultrasonography and Fibroscan ${ }^{\circledR}$ ). 